\section{Calcium in control}

Calcium in Biological Systems. (Symposia of the Society for Experimental Biology.) Edited by C. J. Duncan. Pp. viii +485 . (Cambridge University: Cambridge, London and New York, 1976.) $£ 18$.

THE study of extracellular calcium metabolism and its hormonal control has progressed steadily over many years. Interest in intra-cellular roles of calcium, confined for some time to its regulatory function in muscle, has recently grown rapidly following independent advances in several areas. Calcium is now known to be a key factor in triggering secretion, activating cells for division, controlling intercellular communication and regulating glycolysis and respiration, in addition to its involvement in contractile systems and in nervous conduction.

This latest Symposium of the Society for Experimental Biology considers mainly these intracellular systems. The organisers have concentrated attention on the general problems of how intracellular calcium levels are controlled, rather than on the detailed analysis of the effector roles of $\mathrm{Ca}^{2+}$ which differ greatly in different systems. This is a particularly difficult experimental field for the very reason that calcium is such a good controlling ion; its intracellular concentration is normally in the submicromolar range so that small fluxes have large effects.

It is only within the last five or ten years that the discovery of aequorin by Shimomura and Johnson has provided a direct approach to its measurement. These authors contribute a useful paper on the properties of this interesting luminescent protein and other papers by P. F. Baker and C. C. Ashley et al. describe its application in nerve axons and in muscle fibres. Baker also provides a good general analysis of intracellular $\mathrm{Ca}^{2+}$ regulation, and Ashley et al. consider the technical problems in the quantitative interpretation of aequorin luminescence. They also describe the use of a microscintillator probe to follow the influx of ${ }^{45} \mathrm{Ca}^{2+}$ but they do not evaluate the relative merits of these two techniques.

Two good introductory chapters deal with the general chemistry of $\mathrm{Ca}^{2+}$ and its complexes (R. J. P. Williams) and with a variety of natural and synthetic ionophores for divalent cations (M. Truter). I missed a similar introductory section on the use of electrophysiological techniques and concepts and the uninitiated will find unfamiliar jargon and usage in this field. For example, the significance of a poten- tially interesting paper on the effects of intracellular calcium on membrane permeability (R. W. Meech) was obscured by the use of the term 'activation' in different and undefined senses.

Almost half of the twenty papers concern contractile systems and three of these were on troponin. A clear account of its evolutionary relationships with parvalbumins and myosin light chains, based on amino acid sequences (J. H. Collins), was complemented by a comparison of control functions in different species and a study of the functional interchangeability of these regulatory proteins in vitro (A. Szent-Gyorgyi). It was pointed out (S. Ebashi et al.) that in spite of such homologies one must be cautious about arguing too closely from the regulatory mechanisms in skeletal muscle to other situations, since in smooth muscle, for example, calcium behaves as an activator rather than as a derepressor.

Several papers considered the problems of coupling between excitation and contraction. In skeletal muscle ( $\mathrm{S}$. R. Taylor and E. Godt) a depolarisation induced release from sarcoplasmic reticulum is the main physiological mechanism. The more complex situation in heart muscle is reviewed at the physiological level by $R$. Niedergerke et al., and some of the biochemical problems are discussed by E. Carafoli and $M$. Crompton in their critical evaluation of the role of mitochondria in $\mathrm{Ca}^{2+}$ regulation.

One conclusion to be drawn from several of the contributions is that with the exception of sarcoplasmic reticulum and of $\mathrm{Ca}^{2+}$ uptake by mitochondria, little is known about the nature of intracellular calcium stores and their control. The large reservoir of calcium in the nucleus can best be described as a pool of ignorance.

The conference is ably summarised in a concluding chapter (A. Weber) which also mentions relevant points from the discussion. These tantalising glimpses make one wish that a more extensive record of this part of the meeting could have been included in the book. Nevertheless, the account as it stands provides a valuable survey of a diversifying and developing subject. It will be particularly valuable to workers in the calcium field who wish to keep up-to-date with advances in less familiar parts of their subject.

N. Michael Green

N. Michael Green is a member of the $M R C$ staff, working at the National Institute for Medical Research, London, UK.

\section{By the year 2009 twice as much food, water, energy and mineral ore will be needed to maintain man in his present state of comfort - or discomfort.}

Since the 1973 oil crisis the Western industrialised nations have come to realise that not only oil but almost all resources on which their high standard of living depends are in a finite state of supply, and that the Third World nations are likely to demand a fairer share of these limited resources.

In this book recognised authorities in their fields present a wideranging look at the problems of conservation in terms of future energy needs and energy sources, human energy needs and food supplies, mineral resources and the often ignored areas of fresh air and fresh water.

Paperbound 254pp

$8 \frac{1}{2}^{\prime \prime} \times 5 \frac{1^{\prime \prime}}{8} 085186208 \times £ 6.00$

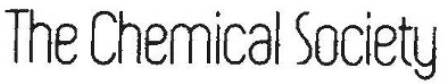

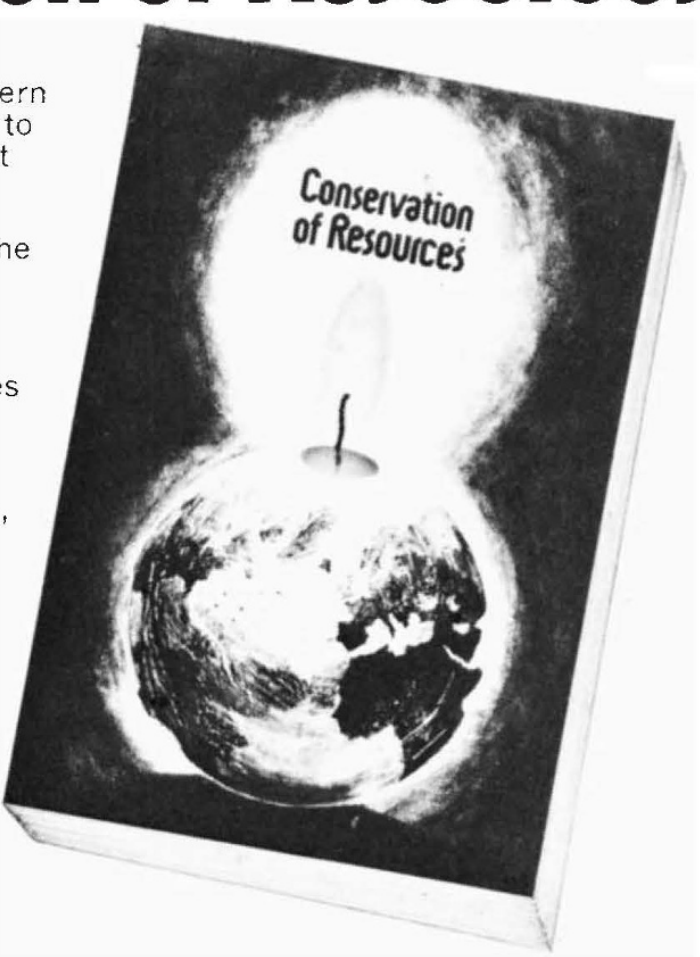

